\title{
The General Movements in children with Down syndrome
}

\author{
Luigi Mazzone, Diego Mugno, Domenico Mazzone* \\ Division of Child Neuropsychiatry, Department of Pediatrics, University of Catania, \\ Via S. Sofia, 78, 95100 Catania, Italy
}

Accepted 28 April 2004

\begin{abstract}
Objective: Aim of our study was to describe the character of General Movements (GMs) in children with Down Syndrome (DS).

Material and methods: GMs of 23 children with DS and of 30 healthy full-term infants were assessed from birth to 6th month corrected age. A qualitative and a semi-quantitative evaluation of GMs were achieved for each child. Data were graphically displayed to obtain growth curves of motor optimality scores.

Results: GMs in children with DS are characterised by low-low/moderate speed, large-large/ moderate amplitude, partially creating impression of fluency, smoothness and complexity, abrupt beginning and end, few other concurrent gross movements. During the 6 months, all children showed an improvement of qualitative and semi-quantitative evaluation, but it was possible to observe great heterogeneity among children in the evolutionary course. GMs evaluation of children with no known motor problems was normal, showing only slight and transient abnormalities at first months. Conclusion: GMs character of children with DS could be related to central nervous system and peripheral abnormalities characterizing this syndrome. The evaluation of GMs in children with DS could be an early marker of motor impairment and help in early management decisions making. (C) 2004 Elsevier Ireland Ltd. All rights reserved.
\end{abstract}

Keywords: General Movements; Children; Down syndrome

\footnotetext{
* Corresponding author. Tel.: +39 95 3782436; fax: +39 95222532.
}

E-mail address: mazzone@unict.it (D. Mazzone). 


\section{Introduction}

Down syndrome (DS), long been recognized as one of the most important causes of disability, is characterized by delays in development of motor function (as a result of associated impairments including muscle hypotonia, joint hyperextensibility, delayed acquisition of postural control, poor balance) and mental retardation [1].

Many brain alterations in children with DS have been described: reduced weight, decreased number and depth of sulci in the cerebral cortex, reduced number of specific populations of neurons, alterations of the cortical laminations, reduced dendritic ramifications, diminished synaptic formations, as well as functional abnormalities in membrane properties [2].

Motor development of children with DS has been studied by many authors primarily by recording the mean age of achievement of motor milestones [3-6]. Chen and Wolley [3], administering the Denver Developmental Screening Test in children with DS, observed that the developmental achievement quotient for the gross motor domain decreases in older children compared to younger; however, the sequence of items passed by children with DS was similar to the sequence of children with no known motor problems.

Russell et al. [7-9] demonstrated a good validity of the gross motor function measure (GMFM) for the assessment of children with DS, and created gross motor function growth curves of children with DS between the ages of 1 month and 6 years.

The aim of our study is to describe the character of General Movements (GMs) in children with DS and to compare it with a control group of healthy full-term infants. Spontaneous motor activity was studied with the Prechtl method, based on the video recording of GMs and the qualitative and semi-quantitative evaluation of GMs.

As far as we know, it is the first study about GMs in children with Down syndrome.

\section{Methods}

The assessment of General Movements (GMs) is a simple, un-invasive and reliable method for neurological evaluation of preterm and term infants. GMs can be observed in fetuses as young as 9 weeks postmenstrual age and after birth until about 4-5 months.

GMs are gross movements, involving the whole body, lasting from a few seconds to several minutes or longer. They wax and wane in intensity, force and speed, and have a gradual beginning and end. Arm, leg, neck and trunk are involved in variable and complex sequences of movements producing the impression of fluency and elegance [10].

During the first 2-month post-term, GMs are commonly referred to as Writhing Movements (WMs). They are characterised by small to moderate amplitude and by slow to moderate speed, and are elliptical in form; this component creates the impression of a writhing quality of movement [11].

At the age of 6 to 9 weeks post-term, the form and character of GMs of typically developing infants changes from a Writhing type to a Fidgety pattern. Fidgety Movements (FMs) are circular movements of small amplitude and moderate speed, they are continual in the awake infant, except during focused attention [12]. They may be concurrent with 
other gross movements, such as kicking, wiggling-oscillating and swiping of the arms or 'pleasure bursts'.

Fidgety movements are transient in healthy infants: initially, they occur as isolated events at about 6 weeks corrected age, gradually increase in frequency, coming to full expression between 9 and 13 weeks corrected age, and then decrease once again between the ages of 14 and 20 weeks post-term corrected age, when infants show other patterns of motor activity, such as manipulation, voluntary movements, reaching, touching, grasping, axial rolling, and antigravity movements [11-14].

The results of GMs evaluation highly correlate with the neurological outcome: a normal quality of GMs consistently predicts a normal neurological outcome, while an abnormal quality of GMs predicts an abnormal outcome.

The method's sensitivity is $94 \%$, and similar in all ages. The specificity is agedependent: it is low during the first 2 months and reaches $82 \%$ to $100 \%$ at 3 -month postterm. There is a robust character of this method: reported inter-observer agreement is between $78 \%$ and $98 \%$, and reported kappa coefficients are between 0.84 and $0.92[15,16]$.

The effects of many neurological and not-neurological diseases on spontaneous motility's character have been analysed in previous studies [17-22].

We performed a prospective study. During the years 1999-2003, 53 children (25 males and 28 females), 23 infants with DS (Down group), 30 healthy full-term infants (control group) were selected.

Down group (13 males and 10 females): all infants had gestational age ranged 36-41 weeks postmenstrual age (mean 38.2 weeks; S.D. 2.76); birth weight between 1759 and $3050 \mathrm{~g}$ (mean $2667.3 \mathrm{~g}$; S.D. 413.13); 13 children had normal delivery, 10 had Caesarian delivery; APGAR score ranged 7-9 at 1 and 5 min. All infants from the Down group had confirmed diagnosis of full trisomy 21 by cytogenetic findings.

Control group (12 males and 18 females): all infants had gestational age ranged 38-40 weeks postmenstrual age (mean 38.9 weeks; S.D. 0.87); birth weight between 2800 and 3400 g (mean 3081 g; S.D. 202.39); normal delivery; APGAR score ranged from 8 to 10 at 1 and $5 \mathrm{~min}$.

Informed consent was obtained for all children who participated to the study. Statistical analysis was performed using the Students' $t$-test.

\subsection{Study design}

For each child, from 1st to 6th month corrected age, we performed an average of two brain ultrasound scans (range 1-3); standardised repeated neurological examination (according to the Dubowitz and Dubowitz [23] protocol during the preterm period and according to the Precthl [24] protocol at term age) and behavioural examinations (according to the Brazelton [25] protocol). Later development was checked by means of the Amiel-Tison and Grenier [26] examination or by use of the Touwen [27] criteria.

\subsection{Observation and evaluation of spontaneous movements}

According to Prechtl $[13,14,28]$, 1-month-old infants were evaluated before discharge from the hospital performing 1 -h videotape recording of the spontaneous motor activity. 
After discharge, 10-30-min videotape recordings were acquired every 4 weeks in the outpatient clinic. All the recordings were made about an hour before feeding and during active wakefulness (States 3-4 according to Prechtl and Beintema [29]). Infants were undressed or partially dressed (i.e., with a body vest and/or nappy), lying supine and unstimulated. The video camera was positioned approximately $1 \mathrm{mt}$ above the cot at an angle of $45^{\circ}$.

During the playback of the video recordings, 3 to 4 GMs each lasting 30 to $120 \mathrm{~s}$, excluding crying, fussing, or suckling periods, were selected as typical for each particular age. A collection of GMs at different ages was then assembled and stored on a separate tape for each infant $[28,30]$.

GMs were analyzed in accordance with the previous studies [21,28,31,32] in two ways: a qualitative assessment of normality or abnormality of GMs, based on a global visual gestalt perception of the complexity, fluency and elegance or monotony and awkwardness of spontaneous movements; and a semi-quantitative estimation of GMs, assessing separate aspects of the spontaneous motor activity: amplitude, speed, sequencing, fluency and elegance, onset and offset.

For the semi-quantitative evaluation of the spontaneous motor activity, we used a modified version (Table 1) of the evaluation sheet used by Ferrari et al. [21].

Table 1

Semi-quantitative estimation of GMs has been assessed evaluating separate aspects of the spontaneous motor activity: amplitude, speed, sequencing, fluency and elegance, onset and offset, movement character

Evaluation sheet of GMs quality

Amplitude

Speed

Sequencing

Fluency and elegance

Onset and offset

$\begin{array}{ll}\text { Predominantly small ranged } & 1 \\ \text { Predominantly large ranged } & 1 \\ \text { Small and large, no intermediate range } & 1 \\ \text { Variable } & 2 \\ \text { Monotonously slow } & 1 \\ \text { Monotonously fast } & 1 \\ \text { Slow and fast, no intermediate } & 1 \\ \text { Invariable } & 1 \\ \text { Variable } & 2 \\ \text { Only synchronized movements } & 1 \\ \text { Disorganized } & 1 \\ \text { Monotonous sequence within single GM } & 1 \\ \text { Some body parts are not involved in the mov. } & 1 \\ \text { Repetition of same sequence from GM to GM } & 1 \\ \text { Variable } & 2 \\ \text { Not fluent, no rotations } & 1 \\ \text { Not fluent, few rotations } & 1 \\ \text { Fluent and elegant, many rotations } & 2 \\ \text { Abrupt } & 1 \\ \text { Minimal fluctuation in intensity } & 1 \\ \text { Smooth crescendo and decrescendo } & 2 \\ & \end{array}$

Total

For each item, we scored 2 points for an optimal performance (indicated in tables with the Italic font stile), and 1 point for a non-optimal one (indicated in tables with the regular font stile). Total score (ranged 5-10) was obtained summing up each item's score. 
According to the optimality concept [33] on each item (Amplitude, Speed, Sequence of movements, Fluency and smoothness, Onset and offset), we scored 2 points for an optimal performance (indicated in tables with the Italic font stile), and 1 point for a non-optimal one (indicated in tables with the regular font stile).

Total score (ranged 5-10) was obtained summing up each item's score.

Qualitative and semi-quantitative assessments of GMs were achieved blindly for all recordings by each of the co-authors. All the observers have a long clinical experience in neurological assessment of preterm and term newborns, and have a 5-year experience with the evaluation of spontaneous motor activity (GMs). The assessors were tested to ensure they had reached acceptable level of inter-rater reliability for scoring of GMs (weighted $k>0.80$ ). Criterion test scores of individual assessors ranged from a kappa value of 0.87 to 0.98 indicating excellent agreement. Gestalt perception was recalibrated each week by reviewing "gold standard" tapes of normal and abnormal GMs $[28,30]$.

Previous studies $[14,19]$ demonstrates that the test-retest reliability after an interval of 2 years is 1.0 for the global judgment (normal vs. abnormal) and 0.85 for the analysis of details (amplitude, movement character, sequencing, fluency and elegance, etc.).

\section{Results}

Normal brain ultrasound scan was found in all children with no known motor problems. Fifteen infants with Down syndrome had normal brain ultrasound scan at birth and eight had transient periventricular hyperecogenicity.

The neurological evaluations in the control group did not show any abnormality; all the children with Down syndrome showed slight to moderate generalised hypotonia.

\subsection{GMs in children with Down syndrome}

\subsubsection{From birth to 2 nd month corrected age}

Qualitative evaluation: GMs were generally characterised by low speed, large amplitude, absence or partial absence of fluency and smoothness; the sequences of arm, leg, neck and trunk movements were generally few variable and complex. No or very few typical elliptical forms of the limb movements were visible. No fast and large extensor movements of the arms or legs were observed. Spontaneous motor activity was scattered during the recording; beginning and end were generally abrupt lacking wax and wane in intensity, force and speed.

Semi-quantitative evaluation: at 1st-month observation, $15 \%$ of children with Down syndrome showed an optimal performance at the parameter "Amplitude" while $85 \%$ had a non-optimal performance; $16 \%$ achieved an optimal performance at the parameter "Speed", while $84 \%$ had a non-optimal one; at the parameters "Onset and offset" and "Fluency and smoothness" $25 \%$ of children with Down children had an optimal performance, while $75 \%$ had a non-optimal one; $60 \%$ showed an optimal performance at the parameter "Sequence of movements", $40 \%$ had a non-optimal performance. Mean total score was 6.69 (S.D. 0.97). 
At 2nd-month observation, the same children improved their motor optimality score: mean total score was 7.56 (S.D. 0.84). In particular, 18\% had an optimal performance at the parameter "Amplitude", 19\% at the parameter "Speed", 27\% at the parameters "Onset and offset", $30 \%$ at the parameter "Fluency and smoothness", and $67 \%$ at the parameter "Sequence of movements".

\subsubsection{From 2nd to 6th month corrected age}

Qualitative evaluation: GMs were characterised by low to moderate speed and large to moderate amplitude, giving a partial impression of fluency and smoothness. The sequences of arm, leg, neck and trunk movements were in almost all children variable and complex. No circular movements of small amplitude and no or very few other gross movements, such as kicking, wiggling-oscillating and swiping of the arms or "pleasure bursts" were observed. GMs were not continual, but scattered during recording. Beginning and end were generally abrupt.

Semi-quantitative evaluation: at 3rd- and 4th-month observations, the mean total score was 7.78 (S.D. 0.99) and at 5th and 6th was 8 (S.D. 0.99).

In particular at 3rd month $20 \%$ of children with Down syndrome showed an optimal performance at the parameter "Amplitude", raising to 25\% at 4th-month observation; $19 \%$ and $25 \%$, respectively, at $3 \mathrm{rd}-$ and 4 th-month observations had an optimal performance at the parameter "Speed"; at 3rd month and then at 4th month $30 \%$ and $32 \%$ had an optimal performance at the parameter "Onset and offset"; $38 \%$ and $40 \%$, respectively, at 3 rd and 4th month showed an optimal performance at the parameter "Fluency and smoothness"; $70 \%$ and then $73 \%$ had an optimal performance at the parameter "Sequence of movements" at 3rd- and 4th-month evaluations.

At 5th and 6th month, percentage of children with DS showing optimal performances was respectively $35 \%$ and $38 \%$ for the parameter "Amplitude", $36 \%$ and $38 \%$ for the parameter "Speed", 35\% and 37\% for the parameter "Onset and offset", 50\% and $62 \%$ for the parameter "Fluency and smoothness", $85 \%$ and $90 \%$ for the parameter "Sequence of movements". Mean total score and S.D. at 5th and 6th month were 8 and 0.6.

Data are summarized in Table 2 and Fig. 1.

\subsubsection{6th-month observation}

We observed a delay in the achievement of manipulation, voluntary and antigravity movements in children with DS; at the age of 6 months, only 7 of the 23 children (30.43\%)

Table 2

Mean scores, standard deviations (S.D.), and $p$ values of children with Down syndrome (DS) and typically developing children (control group) at each time point

\begin{tabular}{llllllcc}
\hline & & 1st month & 2nd month & 3rd month & 4th month & 5th month & 6th month \\
\hline Children with DS & Mean score & 6.69 & 7.56 & 7.78 & 7.78 & 8 & 8 \\
& S.D. & 0.97 & 0.84 & 0.99 & 0.99 & 0.6 & 0.6 \\
Control group & Mean score & 9.26 & 9.73 & 9.9 & 9.9 & 10 & 10 \\
& S.D. & 1.36 & 1.17 & 0.35 & 0.35 & 0 & 0 \\
$p<$ & & 0.0001 & 0.0001 & 0.0001 & 0.0001 & 0.0001 & 0.0001 \\
\hline
\end{tabular}




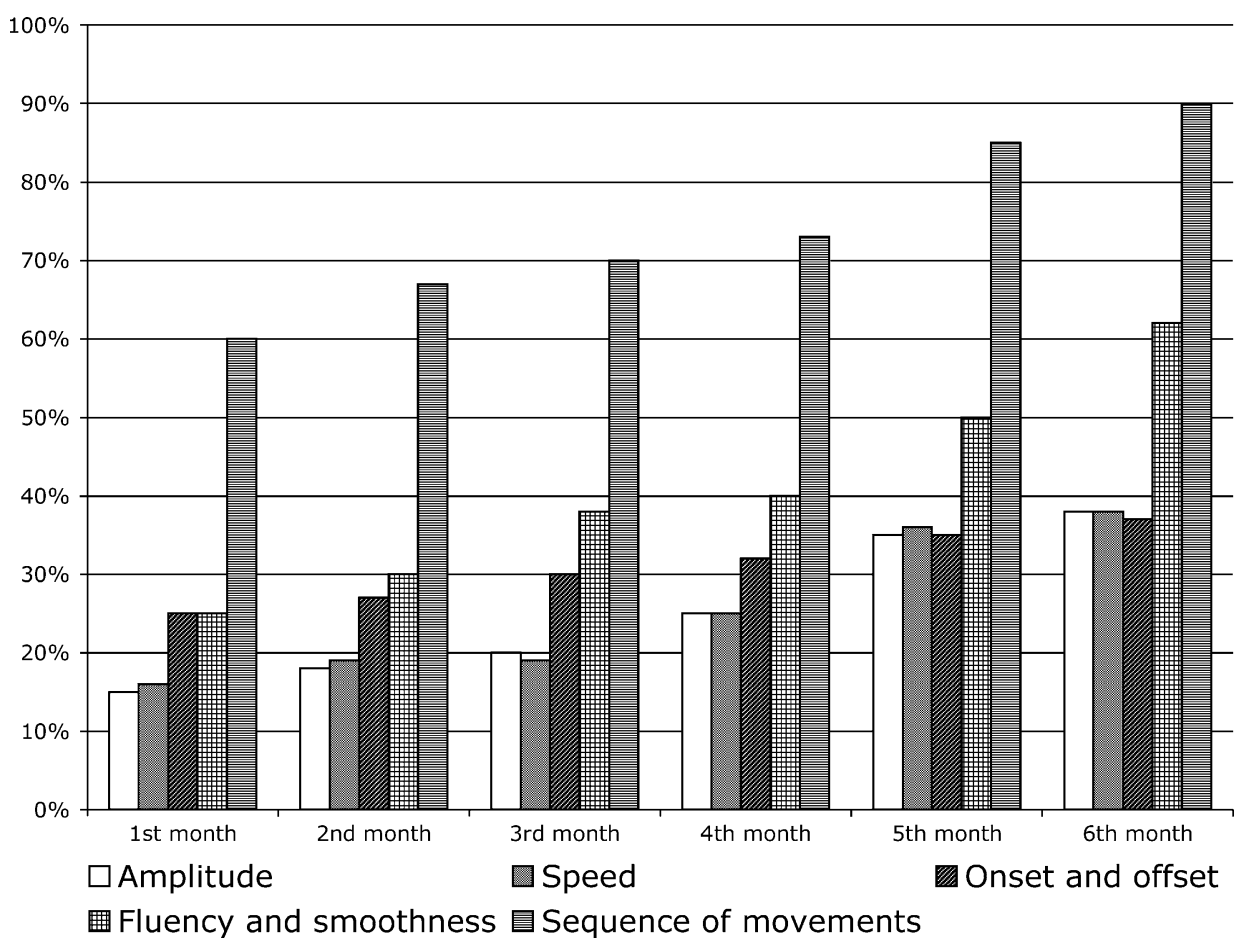

Fig. 1. Children with Down syndrome: for each aspect of GMs (Amplitude, Speed, Onset and offset, Fluency and smoothness, Sequence of movements) percentage of children achieving an optimal performance at each time point is indicated.

showed a decrease in the occurrence of GMs and a clear development of voluntary movements.

\subsection{GMs in control group children}

\subsubsection{From birth to 2 nd month corrected age}

A mean total score of 9.26 (S.D. 1.36) was achieved at first month; five children showed spontaneous motor activity characterised by slight abnormalities (small or large ranged movements, monotonously slow or fast speed, absence of fluency or smoothness), that we considered developmental age-related changes of normal GMs. The sequence of arm, leg, neck and trunk movements were variable and complex in all children.

The 2nd-month evaluation revealed an increase of the mean total scores to 9.73 (S.D. 1.17), GMs giving now the impression of a Writhing quality in almost all infants.

\subsubsection{From 2nd to 6th month corrected age}

All children showed continual circular movements of small amplitude and moderate speed, concurrent with other gross movements (kicking, wiggling-oscillating and pleasure bursts). 


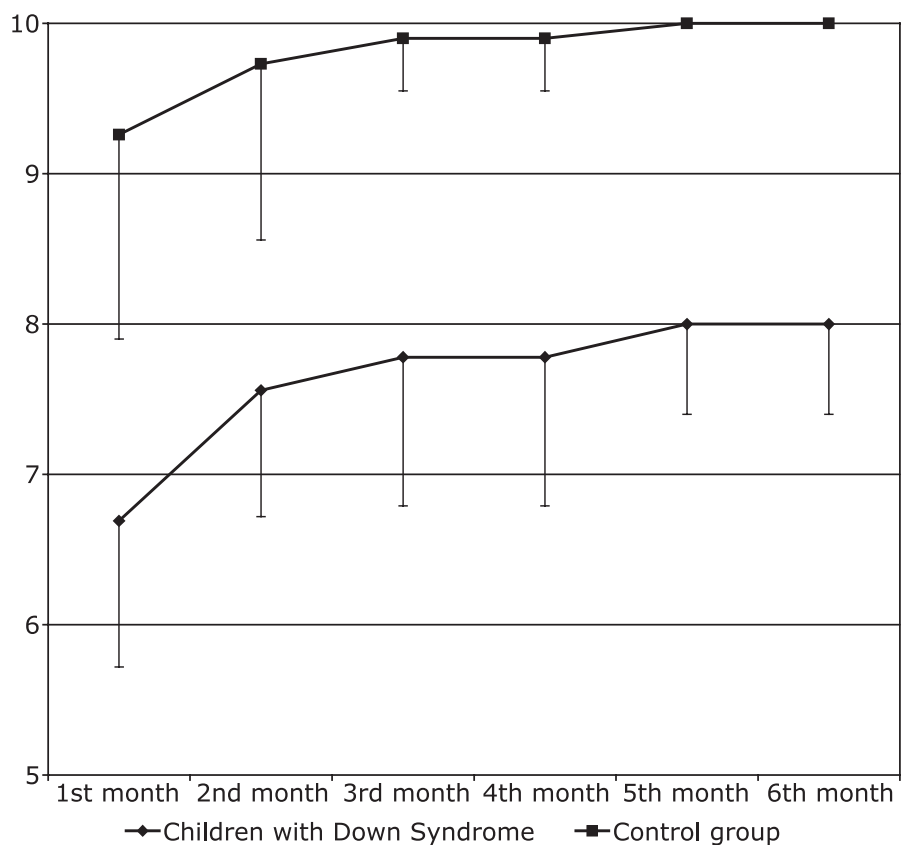

Fig. 2. Mean total scores and standard deviations of the motor optimality score obtained by children with Down syndrome are compared to mean total scores and standard deviations of children with no known motor problems. $p<0.0001$ at each time point.

At 3rd and 4th months, mean total score was 9.9 (S.D. 0.35); increasing to 10 (S.D. 0) at 5 th and 6 th months.

The other patterns of motor activity, such as manipulation, voluntary movements, reaching, touching, grasping, axial rolling, and antigravity movements gradually developed at 15-20 weeks post-term age.

Data from the semi-quantitative evaluation were graphically displayed to obtain growth curves of motor optimality scores (Fig. 2). For each time point, mean total scores and standard deviations of children with Down syndrome were compared to mean total scores and standard deviations of children with no known motor problems (Table 1).

Student's $t$-test demonstrated statistically significant differences between the two groups at each observation $(p<0.0001)$.

\section{Discussion}

The aim of our study was to describe the character of the spontaneous motor activity in children with DS, compared with healthy full-term infants.

Five different patterns of abnormal GMs have been described by Prechtl et al. During the prenatal period and during preterm, term and the first 2 months post-term age: Poor repertoire General Movements (PR), characterised by lack of complexity and variability of sequence of the successive movement components; Cramped-synchronised General 
Movements (CS), characterised by rigid and abrupt movements, and lack of the normal smoothness and fluency; Chaotic General Movements (Ch), characterised by large amplitude of the movements, without any fluency nor smoothness.

Fidgety movements are referred as abnormal if they are Absent $(\mathrm{F}-)$ : FMs are never observed from ages 6 to 20 weeks post-term, or Abnormal $\left(\mathrm{F}_{\mathrm{A}}\right)$ : when their amplitude, speed and jerkiness are moderately or greatly exaggerated $[31,32]$.

Prechtl et al. demonstrated that observation of spontaneous motor activity (GMs) in young infants allows valid predictions about later neurological outcome [14]. The qualitative assessment of GMs during first 2 months (WMs) has an high sensitivity, but its specificity is considerably lower [28]. Many of the earlier GMs abnormalities are transient phenomena and normalise before or at the appearance of Fidgety movements. Abnormal or Absent Fidgety movements are indicative of a poor outcome; Normal Fidgety movements are an excellent marker for a normal neurological outcome [14-16,34].

During first 2 months, GMs of children with Down syndrome are generally characterised by low speed, large amplitude, absence of fluency and smoothness, few variability of limbs movements, absence of small ranged limbs movements and of other gross movements.

During the next months, speed of movement change from low to low-moderate, amplitude change from large to large-moderate, and it is possible to observe small-ranged limbs movements and changes in direction of the movements (as well as other gross movements like kicking, wiggling-oscillating and swiping of the arms) partially creating the impression of fluency, smoothness and complexity.

The typical change in form of GMs recurring at the end of the second and beginning of the third month in children with no known motor problems from Writhing to Fidgety character, has not been observed in children with DS. From birth to 6th-month corrected age, GMs of children with DS become progressively faster, smaller ranged, more fluid and elegant, and sequences of limbs movements more variable and complex, corresponding to a continual and progressive improvement of GMs quality and of the motor optimality score, but it was not possible to observe a clear changing in form (Writhing to Fidgety) of the spontaneous motor activity neither at the end of the 2 nd month nor later.

All children with DS show an improvement of their optimality scores during the observation, but it was possible to observe widely dispersed values of motor optimality scores - as indicated by S.D. - at each time point, reflecting the heterogeneity of these children: mean total optimality score at 1 st month was $6.69 \pm 0.97$; at 2nd month 7.56 \pm 0.84 ; at 3rd and 4th months was 7.78 \pm 0.99 ; at 5 th and 6th months was $8 \pm 0.6$.

The pattern and evolutionary course of GMs in children with DS that we described, as well as the delay in disappearing of the spontaneous motor activity and develop of voluntary movements, can be related to the central nervous system and the peripheral abnormalities that characterize this syndrome. It cannot be considered an abnormal pattern ( $\mathrm{PR}, \mathrm{CS}, \mathrm{Ch}, \mathrm{F}-, \mathrm{F}_{\mathrm{A}}$ ) as observed in children with severe encephalopathy and evolution in cerebral palsy, but has to be considered typical of children with DS.

Palisano et al. administered the GMFM and rated on degree of motor impairment (Mild, Moderate and Severe motor impairment) 133 children with DS to create motor growth curves that describe motor function of children with DS and to compare the rate of 
improvement and upper limit of function between children with mild motor impairment and children with severe or moderate motor impairment.

They demonstrated that on average: (1) Motor impairment has a discernable effect on rate of improvement but only a slight effect on ultimate achievement of gross motor function during early childhood. (2) Children with DS do not achieve all the gross motor functions included on the GMFM by the age of 6 years. (3) Scores improve the fastest at younger ages and then level off as the predicted upper limit of gross motor function is approached $[8,9]$.

The results of studies that have examined motor interventions in children with DS are still inconclusive both for short-term and long-term benefits [4,35-47].

According to Palisano et al., children whose motor function is age appropriate or advanced relative to expectations for children with DS may successfully achieve motor goals through play and structured developmental activities, and intervention may appropriately be directed to other areas of development like occupational therapy. Conversely, children whose gross motor function is delayed relative to expectations for children with DS may be less likely to achieve goals without physical therapy interventions [8].

We believe that it is really important to have assessment instruments that can help professionals to make early management decisions, set goals for intervention and evaluate intervention outcome.

Our hypothesis is that GMs quali-quantitative evaluation could be an early marker of motor impairment, and could have implications for decision about if and when motor interventions are needed.

The growth curves of the motor optimality scores show the average pattern of development of the children with DS (compared to children with no known motor problem) based on cross-sectional data. Children with Down syndrome whose growth curve of motor optimality score is delayed relative to expectation could have a later delay in the rate of improvement of gross motor functions. These information could lead professionals and parents to start an earlier and more intensive physiotherapy intervention, and to set outcomes earlier and more effectively than using other standardized criterionreferenced measures. Further studies are needed to confirm this hypothesis.

\section{References}

[1] Coyle JT, Oster-Granite ML, Gearhart JD. The neurobiologic consequences of Down syndrome. Brain Res Bull 1986 (Jun);16(6):773-87.

[2] Becker L, Mito T, Takashima S, Onodera K. Growth and development of the brain in Down syndrome. Prog Clin Biol Res 1991;373:133-52.

[3] Chen H, Wolley PV. A developmental assessment chart for non-institutionalized Down syndrome children. Growth 1978;42:157-65.

[4] Piper MC, Gosselin C, Gendron M, Mazer B. Developmental profile of Down's syndrome infants receiving early intervention. Child Care Health Dev 1986;12:183-94.

[5] Ramsey M, Piper MC. A comparison of two developmental scales in evaluating infants with Down syndrome. Early Hum Dev 1980;4:89-95.

[6] Cronk C, Crocker A, Pueschel S, Shea A, Zackai E, Pickens G, et al. Growth charts for children with Down syndrome: 1 month to 18 years of age. Pediatrics 1988;81:102-10. 
[7] Russell D, Palisano R, Walter S, Rosenbaum P, Gemus M, Gowland C, et al. Evaluating motor function in children with Down syndrome: validity of the GMFM. Dev Med Child Neurol 1998 (Oct);40(10):693-701.

[8] Palisano RJ, Walter SD, Russell DJ, Rosenbaum PL, Gemus M, Galuppi BE, et al. Gross motor function of children with Down syndrome: creation of motor growth curves. Arch Phys Med Rehabil. 2001 April; 82(4):494-500.

[9] Gemus M, Palisano R, Russell D, Rosenbaum P, Walter SD, Galuppi B, et al. Using the gross motor function measure to evaluate motor development in children with Down syndrome. Phys Occup Ther Pediatr 2001;21(2-3):69-79.

[10] Prechtl HFR. Qualitative changes of spontaneous movements in fetus and preterm infants are a marker of neurological dysfunction. Early Hum Dev 1990;23:151-9.

[11] Hadders-Algra M, Prechtl HFR. Developmental course of GMs in early infancy: I. Descriptive analysis of change in form. Early Hum Dev 1992;28:201-13.

[12] Prechtl HFR, Hopkins B. Developmental transformations of spontaneous movements in early infancy. Early Hum Dev 1986;4:233-8.

[13] Hopkins B, Prechtl HFR. A qualitative approach to the development of movements during early infancy. In: Prechtl HFR, editor. Continuity of neural functions from prenatal to postnatal life. Clin Dev Med, vol. 94. Oxford: Blackwell Scientific Publications; 1984. p. 179-97.

[14] Prechtl HFR, Einspieler C, Cioni G, Bos AF, Ferrari F, Sontheimer D. An early marker for neurological deficits after perinatal brain lesions. Lancet 1997;349:1361-3.

[15] Einspieler C, Prechtl HFR, Ferrari F, Cioni G, Bos AF. The qualitative assessment of general movements in preterm, term and young infants—review of the methodology. Early Hum Dev 1997;50:47-60.

[16] Bos AF. Analysis of movement quality in preterm infants. Eur J Obstet Gynecol Reprod Biol 1998;76: $117-9$.

[17] Bos AF, Martijn A, van Asperen RM, Hadders-Algra M, Okken A, Prechtl HFR. Qualitative assessment of general movements in high risk preterm infants with chronic lung disease requiring dexamethasone therapy. J Pediatr 1998;132:300-6.

[18] Bos AF, van Asperen RM, de Leeuw DM, Prechtl HFR. The influence of septicaemia on spontaneous motility in preterm infants. Early Hum Dev 1997;50:61-70.

[19] Einspieler C. Abnormal spontaneous movements in infants with repeated sleep apnoaes. Early Hum Dev 1994;36:31-49.

[20] Einspieler C, Prechtl HFR, van Eykern L, de Roos B. Observation of movements during sleep in ALTE and apnoeic infants. Early Hum Dev 1994;40:39-50.

[21] Ferrari F, Prechtl HFR, Cioni G, Roversi MF, Einspieler C, Gallo C, et al. Posture, spontaneous movements and behavioural state organisation in infants affected by brain malformation. Early Hum Dev 1997;50:87-113.

[22] Kainer F, Prechtl HFR, Engele H, Einspieler C. Prenatal and postnatal assessment of the quality of general movements in infants of women with type-I diabetes mellitus. Early Hum Dev 1997;50:13-25.

[23] Dubowitz LMS, Dubowitz V. The neurological assessment of the preterm and fullterm newborn infant. CDM, vol. 79. London: Heinemann; 1981.

[24] Prechtl HFR. The neurological examination of the full-term newborn infant. CDM, vol. 63. London: Heinemann; 1977. 2nd rev. ed.

[25] Brazelton TB. Neonatal behavioral assessment scale. CDM, vol. 50. London: Heinemann; 1973.

[26] Amiel-Tison C, Grenier A. Neurologic evaluation of the new-born and term infant. New York: Masson; 1983.

[27] Touwen BCL. Neurological development in infancy. CDM, vol. 58. London: Heinemann; 1976.

[28] Cioni G, Ferrari F, Einspieler C, Paolicelli PB, Barbarini T, Prechtl HFR. Comparison between observation of spontaneous movements and neurological examination in preterm infants. J Pediatr 1997;130:704-11.

[29] Prechtl HFR, Beintema DJ. The neurological examination of the fullterm newborn infant. Clin Dev Med $1964 ; 12: 1-49$.

[30] Cioni G, Ferrari F. Neurological assessment in the first months of life by observation of spontaneous motility. M\&B 1997;10:633-7.

[31] Ferrari F, Cioni G, Prechtl HFR. Qualitative changes of general movements in preterm infants with brain lesions. Early Hum Dev 1990;23:193-233. 
[32] Prechtl HFR, Ferrari F, Cioni G. Predictive value of general movements in asphyxiated fullterm infants. Early Hum Dev 1993;35:91-120.

[33] Prechtl HFR. The optimality concept. Editorial. Early Hum Dev 1980;4:201-5.

[34] Prechtl HFR. State of the art of a new functional assessment of the young nervous system. An early predictor of cerebral palsy. Early Hum Dev 1997;50:1-11.

[35] Hayden AH, Dmitriev V. The multidisciplinary preschool program for Down's syndrome children at the University of Washington model preschool center. In: Friedlander BZ, Sterrit GM, Kirk GE, editors. Exceptional infant: Assessment and intervention, vol. 3. New York: Brunner/Mazel, 1975. p. 193-221.

[36] Connolly B, Russell F. Interdisciplinary early intervention program. Phys Ther 1976;56:155-8.

[37] Aronson M, Fallstrom K. Immediate and long term effects of developmental training in children with Down syndrome. Dev Med Child Neurol 1977;19:489-94.

[38] Hanson MJ, Schwarz RH. Result of a longitudinal intervention program for Down's syndrome infants and their family. Educ Train Ment Retard 1978;13:403-7.

[39] Connolly BH, Morgan S, Russell FF. Evaluation of children with Down syndrome who participated in an early intervention program: second follow-up study. Phys Ther 1984;64:1515-9.

[40] Esenther SE. Developmental coaching of the Down syndrome infant. Am J Occup Ther 1984;38:440-5.

[41] Sharav T, Shlomo L. Stimulation of infants with Down syndrome: long term effect. Ment Retard 1986; 24:81-6.

[42] Brinkworth R. Effects of early home training on the mongol. In: Clark ADB, Clark AM, editors. Mental retardation and behavioural research. Study Group N 4. Edinburgh: Churchill Livingstone, 1972.

[43] Bidder RT, Bryant G, Gray OP. Benefit to Down's syndrome children through training their mothers. Arch Dis Child. 1975 May;50(5):383-386.

[44] Piper MC, Pless IB. Early intervention for children for infants with Down syndrome: a controlled trial. Pediatrics 1980;65:463-8.

[45] Harris SR. Effects of neurodevelopmental therapy on motor performance of infants with Down's syndrome. Dev Med Child Neurol 1981;23:477-83.

[46] Simeonsson RJ, Cooper DH, Scheiner AP. A review and analysis of the effectiveness of early intervention programs. Pediatrics 1982;69:635-41.

[47] Gibson D, Harris A. Aggregated early intervention effects for Down's syndrome persons: patterning and longevity of benefits. J Ment Defic Res 1988;32:1-17. 